\title{
Design and Dynamic Simulation of a Wide-Range Binocular Head-Eye Platform
}

\author{
Jihong Yana , Rufei Cai ${ }^{b}$ \\ State Key Laboratory of Robotics and System Harbin Institute of Technology, Harbin 150000, China \\ ajhyan@hit.edu.cn, brufeicai22@163.com
}

Keywords: Head-Eye Platform, Binocular Mechanism, Wide-Range Object Recognition, Dynamic Simulation.

\begin{abstract}
Binocular head-eye platform is mainly used in the recognition of active objects, and can quickly and flexibly achieve a wide-range image information collection. The existing binocular head-eye platform usually uses small camera with fixed focus lens to obtain image information, so there are some problems, such as close target distance, narrow view, small motion range and etc. For these reasons, this paper presents a new type of binocular head-eye platform. The 7-dof head-eye platform has long target distance, wide-range view, large motion range and high speed performance for zoom lens camera and series parallel hybrid mechanism. Monocular mechanism has enough stiffness to ensure the high-speed movement of heavy payload, due to the parallel design. For motion control, kinematics solution is solved by DH parameter method and analytical method. DH parameter method is easy to establish the coordinate transformation relation. And analytical method can obtain accurate mathematical expressions. To verify the rationality and feasibility of the platform, dynamic simulation is carried out by software ADAMS, while achieving its performance specification in three axes.
\end{abstract}

\section{Introduction}

Head-eye platform is mainly used in the field of active vision ${ }^{[1-2]}$. At present, the design method is to use small camera with fixed focus lens to obtain image information, so there are some problems, such as close target distance, narrow view, small motion range and so on.

The camera control is realized by serial mechanism in traditional way. The most representative is the Yorick series head-eye platforms ${ }^{[3]}$, in which the Yorick $811 \mathrm{R}$ prototype has 4 degrees of freedom, which are head yaw and pitch, as well as binocular yaw. Special DC servo motor and position sensor make it small and compact. Icub head-eye platform ${ }^{[4-5]}$ also uses a similar mechanism with 6 degrees of freedom, but changes the independent pitch of both cameras to public pitch. Domestic colleges and universities have also developed a number of head-eye platform prototypes. In 2004, double CCD humanoid head-eye platform ${ }^{[6]}$ was developed in Zhejiang University. In 2005, Yangzhou University made a 4-dof active vision head-eye platform ${ }^{[7]}$ etc.

Monocular mechanism is an important part of the head-eye platform, and its design is usually divided into two ways: series and parallel. The series is easy to realize and the motion space is large. However, it is difficult to achieve the effect of high speed and high stiffness due to the error accumulation and the increase of load inertia. Parallel mechanism can effectively avoid the above defects, but it also brings problems of small motion range and complicated control. The design of active vision platform is limited by the size of motor, reducer and camera. In most systems, the motor will increase the rotational inertia of the moving load. The design method of the parallel mechanism, such as agile eye ${ }^{[8]}$ and CeDAR ${ }^{[9]}$, can effectively avoid the problem and achieve high speed and high stiffness.

The traditional head-eye platform load object is small camera with fixed focus lens, which causes disadvantages of close target distance, narrow view, small motion range and so on. In order to solve the above problems, this paper presents a novel series parallel hybrid head-eye platform, which is suitable for ordinary camera with zoom lens. At first, introduce the mechanism design. Then use 
analytical method and DH parameter method to solve kinematics problem. Finally, analyze dynamic performance by ADAMS simulation.

\section{Mechanism Design}

Binocular head-eye platform for long distance, wide vision field and target recognition, not only requires agencies to achieve large-scale image information acquisition, but also takes motion to adjust the speed, stability and reliability.

\subsection{Design and Parameters.}

Fig. 1 is the three-dimensional model of the head-eye platform. The model is composed of two monocular mechanisms and a neck mechanism. The overall size is about 400x200x360mm. Two monocular mechanisms symmetrically form a binocular system. Monocular mechanisms and neck mechanism are connected by cantilevers to prevent the interference and reduce the inertia of load. The distance between two camera axes is defined as the baseline distance, which is much larger than that of normal human eyes (about $70 \mathrm{~mm}$ ). The baseline distance can be reduced effectively by reducing the range of motion or selecting small camera.

Tab.1 is the parameters of head_eye platform. Angle range, angular velocity and acceleration are reference normal human head and eye movement parameters. The angle range is larger than normal range of eye movements to obtain greater vision field. The payload is the overall weight of zoom lens and camera. And zoom lens focal length range is about $8-80 \mathrm{~mm}$ for wide-range object recognition.

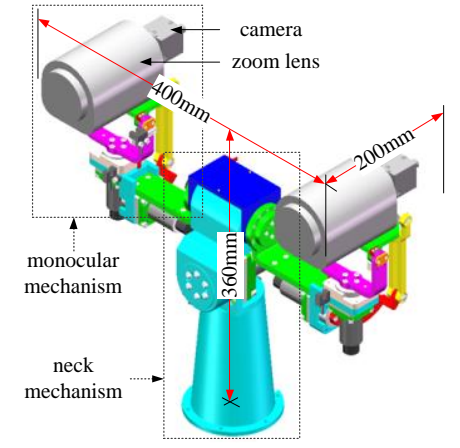

Fig. 1 Design of head-eye platform
Table 1 Specification for joints of head-eye platform

\begin{tabular}{cccc}
\hline & ${\text { Range }\left[{ }^{\circ}\right]}^{\circ}$ & Max Vel $\left[{ }^{\circ} \mathrm{s}-1\right]$ & Max Acc [ $\left.{ }^{\circ} \mathrm{s}-2\right]$ \\
\hline Neck tilt & \pm 90 & 500 & 2500 \\
Neck swing & \pm 45 & 500 & 2780 \\
Neck pan & \pm 90 & 500 & 2500 \\
Eye tilt & \pm 60 & 500 & 2500 \\
Eyes pan & \pm 60 & 500 & 2500 \\
Payload & \multicolumn{3}{|}{$800 \mathrm{~g}$} \\
Focal length & \multicolumn{3}{|c}{$80 \mathrm{~mm}$} \\
\hline
\end{tabular}

\subsection{Structure Composition.}

Neck mechanism, as shown in Fig.2, is a 3-dof series robot, which can pan, swing and tilt. The three joints are stacked in turn with the axes orthogonal to each other, and each joint is designed by encoder, motor and reducer, which are arranged in the coaxial line. The design is simple, easy to control and meets all required performance.

There are two typical configurations for binocular structure: Holmholtz and Fick ${ }^{[10]}$. The former has a common pitch axis, which can not completely imitate the opposite direction motion of human eyes. Considering the practical application, the platform uses the Fick configuration.

Fig. 3 and Fig.4 show the monocular mechanism. The monocular mechanism is a 2-dof parallel turntable, which can realize the motion of the camera around the horizontal axis and the vertical axis. The parallel mechanism consists of moving platform, two moving branches and fixed platform. The payload is a zoom lens and an ordinary camera, which is installed on the moving platform, and the yawing branch chain is driven directly by motor after connecting with reducer. The connecting rod on both sides of the double end support, reduce the axial error caused by bearing clearance, while the mechanism of force stability. Active rod with curved design meets the motion while ensuring that the organization is compact. The speed ratio of 100:1 harmonic reducer increase torque and make sure the transmission accuracy. The mechanism can realize high speed movement of payload in the range of tilt and pan 60 degrees. 


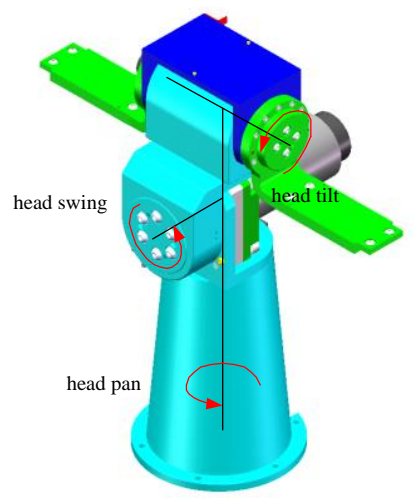

Fig. 2 Neck mechanism

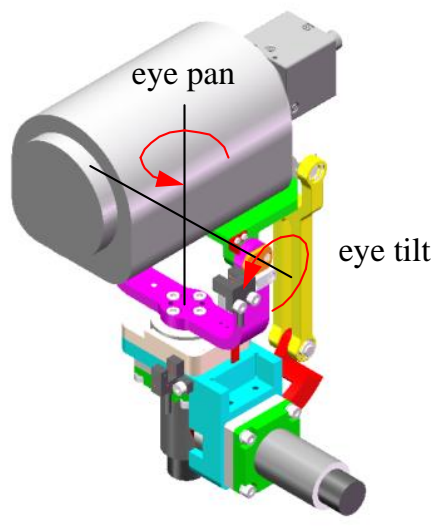

Fig. 3 Monocular mechanism

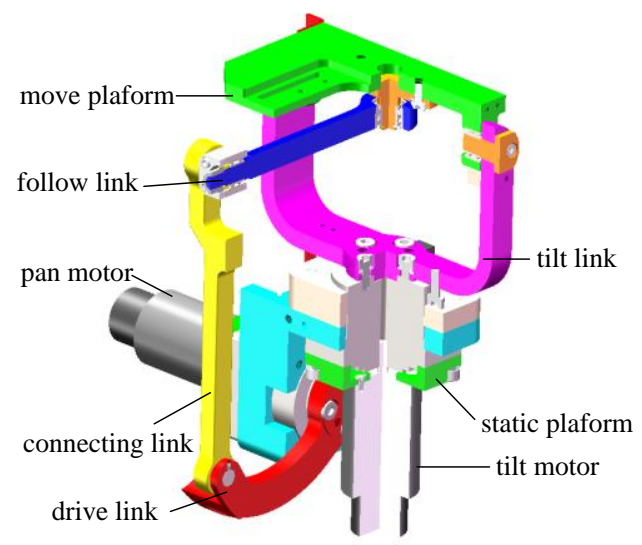

Fig. 4 Profile for monocular mechanism

\section{Kinematic Analysis}

In order to realize the motion control, it is necessary to establish the relationship between the rotation angle of the joint and the rotation of the end. As the head-eye platform is a series parallel hybrid mechanism, this paper uses analytic method and DH parameter method to solve its kinematics solution.

\subsection{Head-Eye Platform Analysis.}

DH parameter method is a common method for kinematic analysis of series robot. It uses a homogeneous transformation matrix to realize the conversion between the terminal coordinate system and the base coordinate system.

The coordinate systems and variables of the head-eye platform are shown in Fig.5. The corresponding DH parameters are shown in Tab.2 The homogeneous transformation matrix of the coordinate system between the $\operatorname{rod} i-1$ and the $\operatorname{rod} i$ is as follows

$$
{ }^{i-1} A_{i}=\operatorname{Rot}\left(z_{i-1}, \theta_{i}\right) \operatorname{Tran}\left(z_{i-1}, d_{i}\right) \operatorname{Tran}\left(x_{i}, a_{i}\right) \operatorname{Rot}\left(x_{i}, \alpha_{i}\right)
$$

The coordinate system $\sum 0$ is transformed into the coordinate system $\sum 5 l$ and $\sum 5 r$ by the homogeneous transformation matrix.

$$
\begin{aligned}
& { }^{0} T_{5 l}={ }^{0} A_{1}{ }^{1} A_{2}{ }^{2} A_{3 l}{ }^{3 l} A_{4 l}{ }^{4 l} A_{5 l} \\
& { }^{0} T_{5 r}={ }^{0} A_{1}{ }^{1} A_{2}{ }^{2} A_{3 r}{ }^{3 r} A_{4 r}{ }^{4 r} A_{5 r}
\end{aligned}
$$

In the above solving process, $\theta_{4 l}, \theta_{5 l}, \theta_{4 r}$ and $\theta_{5 r}$ are intermediate variables.

$$
\theta_{4 l}=q_{1 l}, \theta_{5 l}=q_{2 l}, \theta_{4 r}=q_{1 r}, \theta_{5 r}=q_{2 r}
$$

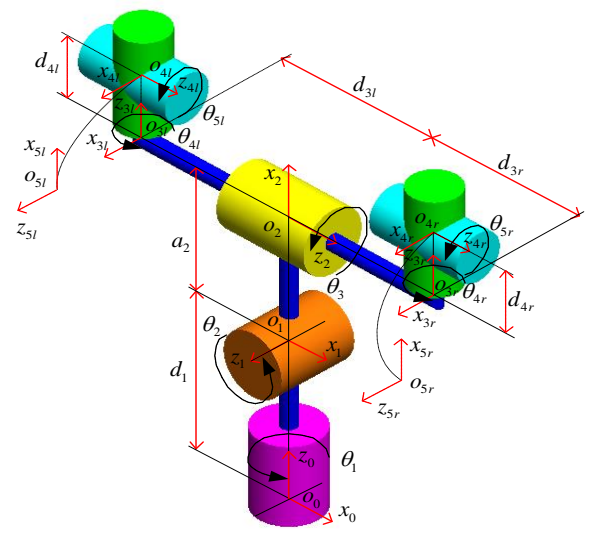

Table $2 \mathrm{DH}$ parameters

Fig. 5 Head-eye platform coordinate system

\begin{tabular}{ccccc}
\hline Jiont $i$ & $\theta_{i}$ & $\alpha_{i}$ & $a_{i}$ & $d_{i}$ \\
\hline 1 & $\theta_{1}=0$ & $90^{\circ}$ & 0 & $d_{1}$ \\
2 & $\theta_{2}=90^{\circ}$ & $90^{\circ}$ & $a_{2}$ & 0 \\
31 & $\theta_{3}=90^{\circ}$ & 0 & 0 & $-d_{3 l}$ \\
41 & $\theta_{4 l}=0$ & $-90^{\circ}$ & 0 & $d_{4 l}$ \\
51 & $\theta_{5 l}=-90^{\circ}$ & $-90^{\circ}$ & 0 & 0 \\
$3 \mathrm{r}$ & $\theta_{3}=90^{\circ}$ & 0 & 0 & $d_{3 r}$ \\
$4 \mathrm{r}$ & $\theta_{4 r}=0$ & $-90^{\circ}$ & 0 & $d_{4 r}$ \\
$5 \mathrm{r}$ & $\theta_{5 r}=-90^{\circ}$ & $-90^{\circ}$ & 0 & 0 \\
\hline
\end{tabular}

The values of the process are solved by monocular mechanism kinematics analysis. So the forward kinematics is solved. 
When the homogeneous transformation matrix of the end coordinate system ${ }^{0} T_{5 l}$ or ${ }^{0} T_{5 r}$ is known, the variable $\theta_{1}$ can be solved by ${ }^{0} A_{1}^{-10} T_{5 l}={ }^{1} A_{2}{ }^{2} A_{3 l}{ }^{3 l} A_{4 l}{ }^{4 l} A_{5 l}$. Similarly, other variables can be solved. So the inverse kinematics is solved.

\subsection{Monocular Mechanism Analysis.}

Monocular motion consists of positive solution and inverse solution. From the mathematical point of view, the kinematics of the monocular mechanism is to establish the relationship between the joint angle $\theta\left(\theta_{4}, \theta_{5}\right)$ and the end angle $q\left(q_{1}, q_{2}\right)$. The positive solution is $q=f(\theta)$, and the inverse solution is called $\theta=f^{-1}(q)$.

According to the 3D model of the mechanism:

1) Bars are rigid, not considering the deformation factors.

2) The mechanism can be simplified as Figure 6 and the corresponding coordinate system is set up: the coordinate system $o-x y z$ is fixed on the rod 2, and the origin is located at the center of the intersection between the joint $\mathrm{A}$ and the joint $\mathrm{B}$ axis.

3) The joint angle range is $\theta_{4}, \theta_{5} \in\left(-90^{\circ}, 90^{\circ}\right)$, and the initial position shows in Fig.6 The four bar mechanism is a parallel four bar mechanism, so the angle of the rod 3 around the $\mathrm{G}$ axis is equal to the tilt angle $\theta_{5}$.
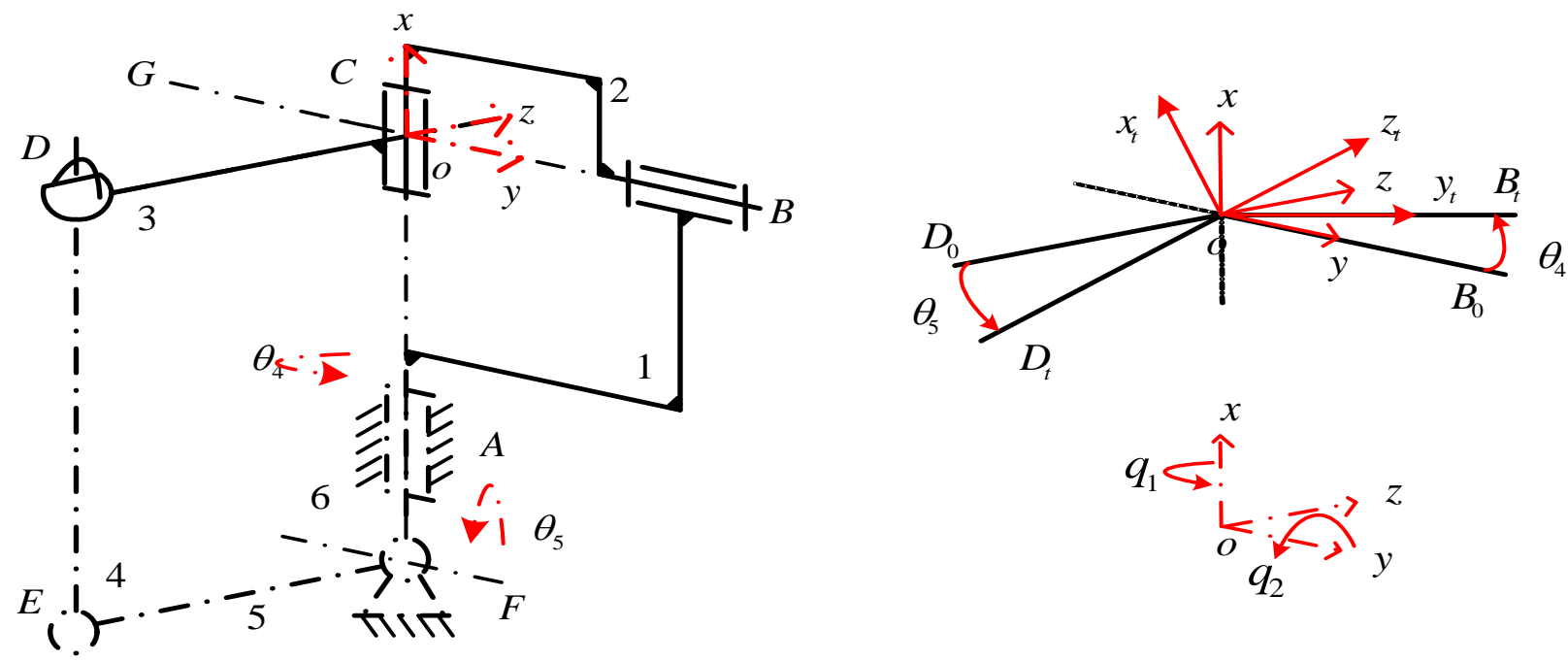

Fig. 6 Diagram of mechanism motion

From 0 time to $t$ moment, joint $B$ axis moves from $B_{0}$ to $B_{t}$, and joint $D$ axis also moves from $D_{0}$ to $D_{t}$. According to the geometric relation of the joint axis : $\overrightarrow{O B_{t}} \perp \vec{e}_{x_{t}} \perp \overrightarrow{O D}$, the unit vector $\overrightarrow{e_{x_{t}}}=\left(a_{x}, b_{x}, c_{x}\right)$ of the $x_{t}$ axis in coordinate system $o-x y z$ can be obtained.

$$
\left\{\begin{array}{c}
\overrightarrow{O D}_{t}=\left(-\sin \theta_{5}, 0,-\cos \theta_{5}\right) \\
\overrightarrow{O B}_{t}=\left(0, \cos \theta_{4}, \sin \theta_{4}\right) \\
\left|\overrightarrow{e_{x_{t}}}\right|=\sqrt{\left(a_{x}^{2}+b_{x}^{2}+c_{x}^{2}\right)}=1 \\
\overrightarrow{O D_{t}} \bullet \overrightarrow{e_{x_{t}}}=\overrightarrow{O B_{t}} \bullet \overrightarrow{e_{x_{t}}}=0
\end{array}\right.
$$

Eliminate 


$$
\left\{\begin{array}{c}
a_{x}=1 / m \\
b_{x}=\left(\tan \theta_{4} \tan \theta_{5}\right) / m \\
c_{x}=-\tan \theta_{5} / m
\end{array}\right.
$$

For the formula $m= \pm \sqrt{1+\tan ^{2} \theta_{5}+\tan ^{2} \theta_{4} \tan ^{2} \theta_{5}}$, the initial position can be determined from the positive solution.

The unit vector of the $y_{t}$ axis is the same as the vector $\overrightarrow{O B}_{t}$ direction, and then the unit vector of the $z_{t}$ axis in the coordinate system $o-x y z$ can be calculated.

$$
\left\{\begin{array}{c}
\overrightarrow{e_{x_{t}}}=\left(a_{x}, b_{x}, c_{x}\right) \\
\overrightarrow{e_{y_{t}}}=\left(0, \cos \theta_{4}, \sin \theta_{4}\right) \\
\left|\overrightarrow{e_{z_{t}}}\right|=\sqrt{\left(a_{z}^{2}+b_{z}^{2}+c_{z}^{2}\right)}=1 \\
\overrightarrow{e_{x_{t}}} \bullet \overrightarrow{e_{z_{t}}}=\overrightarrow{e_{y_{t}}} \bullet \overrightarrow{e_{z_{t}}}=0
\end{array}\right.
$$

Eliminate

$$
\left\{\begin{array}{c}
a_{z}=\left(b_{x} \tan \left(\theta_{4}\right)+c_{x}\right) /\left(a_{x} n\right) \\
b_{z}=-\tan \left(\theta_{4}\right) / n \\
c_{z}=1 / n
\end{array}\right.
$$

For the formula $n= \pm \sqrt{1+\tan ^{2}\left(\theta_{4}\right)+\left(b_{x} \tan \left(\theta_{4}\right)+c_{x}\right)^{2} / a_{x}^{2}}$, the initial position can be determined from the positive solution.

Combined type (4) and (5), the transformation matrix of coordinate system between $o-x y z$ and $o_{t}-x_{t} y_{t} z_{t}$ can be obtained.

$$
T_{\theta}=\left[\begin{array}{ccc}
a_{x} & 0 & a_{z} \\
b_{x} & \cos \left(\theta_{4}\right) & b_{z} \\
c_{x} & \sin \left(\theta_{4}\right) & c_{z}
\end{array}\right]
$$

The rotation transformation matrix of coordinate system $o-x y z$ to $o_{t}-x_{t} y_{t} z_{t}$ can also be determined by angle $q_{1}$ and $q_{2}$.

$$
T_{q}=\operatorname{Rot}\left(x, q_{1}\right) \operatorname{Rot}\left(y^{\prime}, q_{2}\right)=\left[\begin{array}{ccc}
\cos \left(q_{2}\right) & 0 & \sin \left(q_{2}\right) \\
\sin \left(q_{1}\right) \sin \left(q_{2}\right) & \cos q_{1} & -\sin \left(q_{1}\right) \cos \left(q_{2}\right) \\
-\cos \left(q_{1}\right) \sin \left(q_{2}\right) & \sin q_{1} & \cos \left(q_{1}\right) \cos \left(q_{2}\right)
\end{array}\right]
$$

Obviously $T_{\theta}=T_{q}$, the corresponding elements are equal.

$$
\left\{\begin{array}{c}
q_{1}=-\theta_{4} \\
q_{2}=-\arctan \left(\tan \left(\theta_{5}\right) * \sqrt{1+\tan ^{2}\left(\theta_{4}\right)}\right)
\end{array}\right.
$$

The following conclusions can be drawn from the formula (10):

1) Pan angle $q_{1}$ is determined entirely by the joint $A$ angle $\theta_{4}$. And when $\theta_{4}=0$, the tilt angle $q_{2}$ is determined solely by the joint $F$ angle $\theta_{5}$.

2) When $\theta_{5} \neq 0$, the tilt angle $q_{2}$ has strong coupling characteristics, which is determined by joint angle $\theta_{4}$ and $\theta_{5}$.

The inverse solution can be obtained by solving the inverse equation (10). 


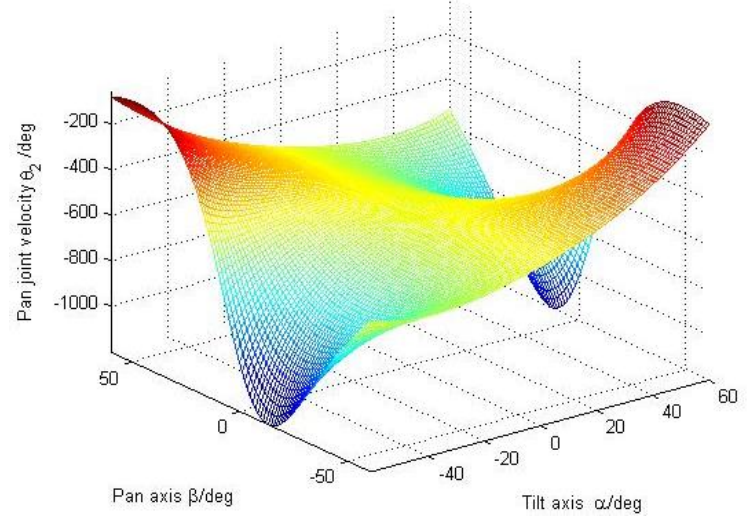

Fig. $7 \dot{q}_{1}=0^{\circ} / s, \dot{q}_{2}=500^{\circ} / s$, expected angular velocity

$$
\left\{\begin{array}{c}
\theta_{4}=-q_{1} \\
\theta_{5}=-\arctan \left(\tan \left(q_{2}\right) / \sqrt{1+\tan ^{2}\left(q_{1}\right)}\right)
\end{array}\right.
$$

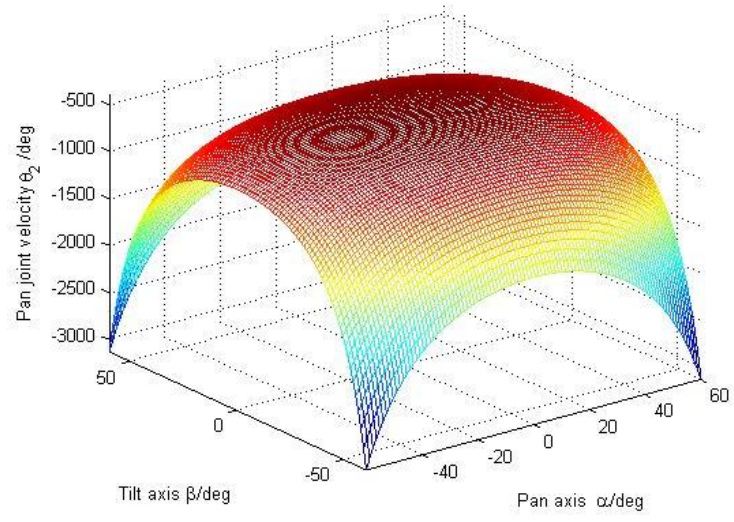

Fig. $8 \dot{q}_{1}=500^{\circ} / s, \dot{q}_{2}=500^{\circ} / s$, expected angular velocity

Formula (10) derivation of time is the angular velocity expression. It is obvious that the tilt angular velocity is related to the position and velocity of the joint, and the pan velocity is determined only by the pan velocity.

As shown in Fig.7, indicates the expected tilt angular velocity of the entire workspace, when the camera rotates around the tilt axis at a speed of $500 \% \mathrm{~s}$ and the pan axis remains stationary. The maximum expected tilting angular velocity is about $1200^{\circ} / \mathrm{s}$, and is opposite to the tilt angle velocity.

As shown in Fig.8, indicates the desired tilt angular velocity of the entire workspace, when the camera rotates around the tilt and pan axes at a speed of $500 \%$. The maximum expected tilting angular velocity is about $3000 \%$, which occurs at the corner of the camera.

\section{Dynamic Simulation Analysis}

In order to investigate the maximum output torque of each joint and the rationality and feasibility of the design, the ADAMS software was used to simulate the dynamics.

The model is imported into ADAMS software. First it needs to simplify and remove fasteners. Then add materials for each component. Finally add constraint, drive and gravity environment. The whole process of simulation is without considering the friction. During the whole process of the head-eye platform, the swing joint of the neck mechanism has the biggest torque, so the joint is selected as the object of the simulation study. Fig.9 is the simulation of the movement of the neck mechanism.

Fig. 10 shows the input angle, angular velocity and angular acceleration of the swing joint. Solid line, dotted line and point line are respectively used to express the angle, angular velocity and angular acceleration curve. From the left position -45 degree to the right position 45 degree, swing joint is first accelerated to the maximum speed $\left(500^{\circ} / \mathrm{s}\right)$ and then decelerated to rest.

Fig. 11 shows the output torque curve of swing joint during the whole movement. The maximum output torque of the joint occurs in the limit position, its absolute value is about $4937 \mathrm{mNm}$. The torque is the output value of the harmonic reducer, and the reducer speed ratio is 100:1, so the maximum output torque of the motor shaft is about $50 \mathrm{mNm}$. This torque can be used as the basis for motor selection. The sudden change of the joint torque curve is caused by the torque sampling points.

The second simulation object is the tilt and pan joint of the monocular mechanism. The simulation process is shown in Fig. 12. The right camera tilts from the upper limit of the position -60 degree to the lower limit 60 degree, while the left camera pans angle from the left limit position -60 degree to the right position 60 degree.

Fig. 13 shows the input angle, angular velocity and angular acceleration curve of the tilt joint of the right monocular mechanism. Solid line, dotted line and point line are respectively used to express the angle, angular velocity and angular acceleration curve. During the whole movement, each joint first 
accelerates to the maximum angular velocity, then keeps uniform motion, at last decelerates and stops at the limit position.
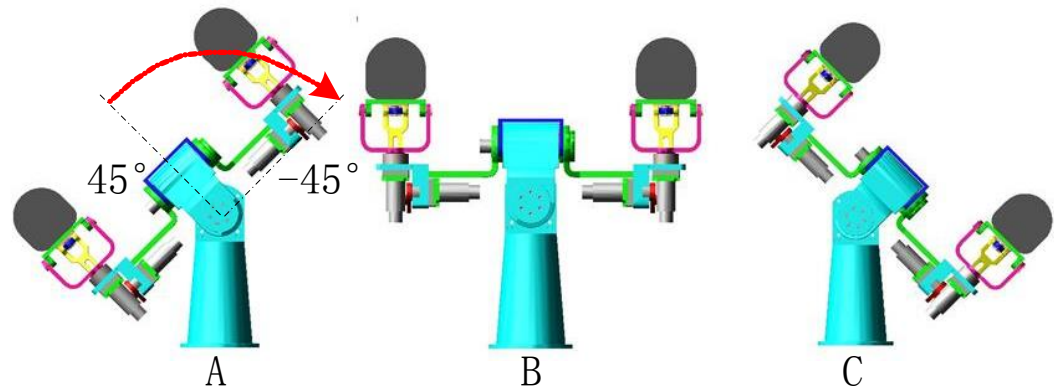

Fig. 9 Simulation of neck mechanism swing joint

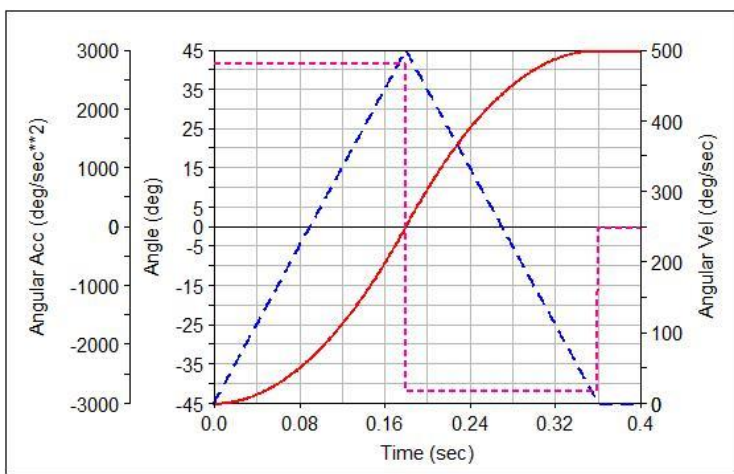

Fig. 10 Input angle, angular velocity and angular acceleration of swing joint

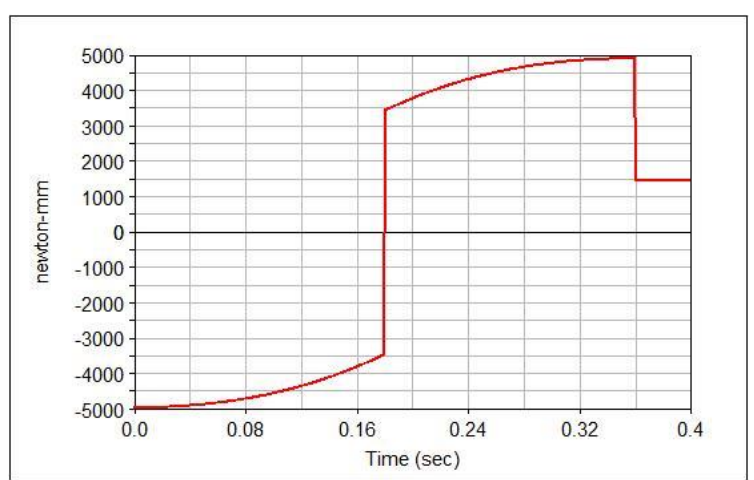

Fig. 11 Output torque curve of swing joint

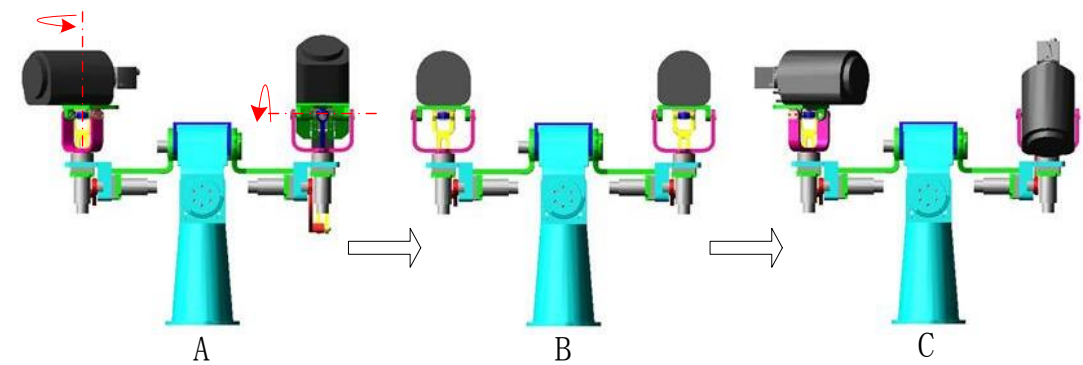

Fig. 12 Simulation of the joint action of the monocular mechanism

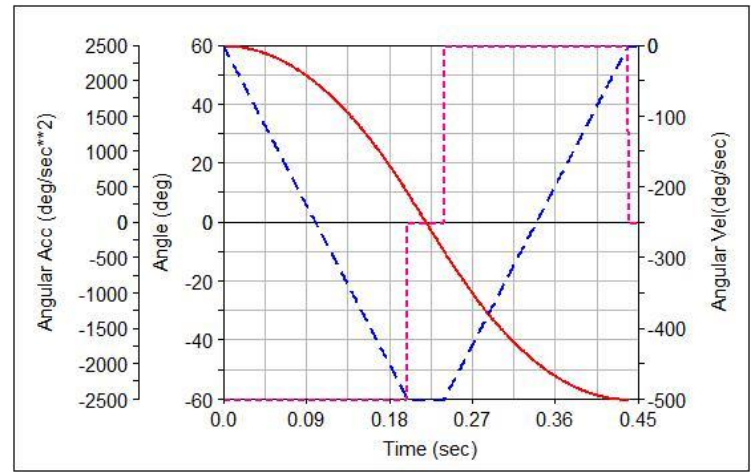

Fig. 13 Input angle, angular velocity and

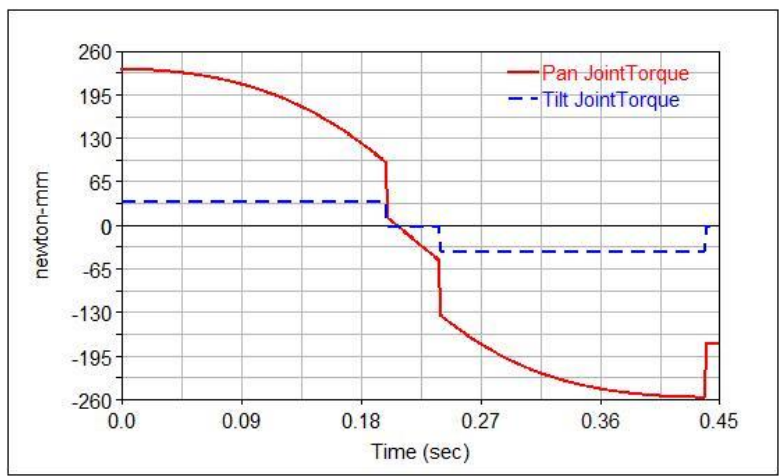

Fig. 14 Output torque curve of joints angular acceleration curve of joints

Fig. 14 shows the output torque curve of the tilting and pan joints of the monocular mechanism during the whole movement. Obviously, the maximum output torque of pan joint (about $37 \mathrm{mNm}$ ) is far less than the maximum output torque of tilting joint (about $255 \mathrm{mNm}$ ), which is about $1 / 6$. The main reason is that the tilting joint needs to overcome the combined effect of inertia torque and gravity torque, and gravity torque is much larger than inertia torque. 


\section{Conclusion}

In this paper, a new series parallel hybrid binocular head platform is introduced, which is applied to the active vision of wide range and long distance object recognition.

1) The special design has high speed and large range. Zoom lens and camera are used to realize the remote object recognition. Achieve high speed tilting and pan motion by parallel mechanism and the range is about two times the normal human eye. Enlarge binocular vision with serial neck mechanism.

2) Use DH parameter method to solve the coordinate transformation of head-eye platform. Solved monocular mechanism kinematic solution by analytical method, and then analyze the expected speed of the monocular tilting joint in the whole workspace.

3) Carry out dynamic simulation in ADAMS to analyze the torque output curves of swing joint, monocular tilting joint and pan joint. The torques can be used as the basis for motor selection.

\section{Acknowledgments}

This work was supported in part by National High-tech R\&D Program of China (No. 2015AA042306).

\section{References}

[1]. ZHU Junjie, WANG Bin, LIU Hong, et al. Design and Object Tracking Research of a Humanoid Robot Head [J]. Machinery and Electronics, 2010, 2010 (7): 49-52.

[2]. ZHANG Xiao-jun, ZHANG Ming-lu, DAI Shi-jie. Survey on humanoid robot heads [J].Journal of Instrumentation, 2009 (1): 219-224.

[3]. Murray D W, McLauchlan P F, Reid I D, et al. Reactions to peripheral image motion using a head/eye platform[C]//Computer Vision, 1993. Proceedings. Fourth International Conference on. IEEE, 1993: 403-411.

[4]. Beira R, Lopes M, Praça M, et al. Design of the robot-cub (icub) head [C]//Robotics and Automation, 2006. ICRA 2006. Proceedings 2006 IEEE International Conference on. IEEE, 2006: 94-100.

[5]. Rodríguez N E N. Design issue of a new iCub head sub-system [J]. Robotics and Computer-Integrated Manufacturing, 2010, 26(2): 119-129.

[6]. Pan-feng. Study on theory and application of human's eye and neck vision simulation system [D]. Hangzhou: Zhejiang University, 2005.

[7]. ZHU Xing-long, ZHOU Ji-ping. Design on binocular stereovision apparatus with laser token [J]. Machinery Manufacturing and Automation, 2005, 34 (4): 129-131.

[8]. Gosselin C M, Pierre E S, Gagne M. On the development of the agile eye [J]. IEEE Robotics \& Automation Magazine, 1996, 3(4): 29-37.

[9]. Truong $\mathrm{H}$, Abdallah S, Rougeaux S, et al. A novel mechanism for stereo active vision [C]//Australian conf. on robotics and automation. 2000.

[10]. Murray D W, Du F, McLauchlan P F, et al. Design of stereo heads [J]. Artificial Intelligence, 1992: 155-172. 